$\xi=-1$

\title{
Stationary wavelet transform based radiometric error correction technique for NOAA-AVHRR sensor data
}

\author{
V. Raja Rajeswari ${ }^{1 *}$, S. Narayana Reddy ${ }^{2}$, P. Jagadamba $^{3}$ \\ ${ }^{1}$ Research Scholar, Sri Venkateswara University College of Engineering, S.V. University, Tirupati, A.P., India \\ ${ }^{2}$ Professor, Sri Venkateswara University College of Engineering, S.V. University, Tirupati, A.P., India \\ ${ }^{3}$ Assistant Professor, SKIT, Sri Kalahasthi, Tirupati, Andhra Pradesh, India-517502. \\ *Corresponding author E-mail:rajeswari.spmvv@gmail.com
}

\begin{abstract}
Due to the inaccuracy of the sensing devices, remote sensing images contain radiometric errors, which can be severe in many cases. Therefore, the preprocessing is an inevitable step in the remote sensing image analysis. This paper presents radiometric errors and evaluates methodologies to retrieve information contained in images by means of filtering in the spatial domain and wavelet domain. Among those, the wavelet techniques are more effective to reduce noise because of their ability to capture the energy of a signal in fewer wavelet coefficients. In this study, Stationary Wavelet Transform (SWT) method and its application to NOAA -18, 19 AVHRR/3 channel 3 and channel 4 images to correct radiometric error is presented. Qualitative and quantitative analysis was carried to evaluate the performance of SWT method, both by measuring the Peak Signal to Noise Ratio (PSNR) and Structural Similarity Index Measure (SSIM), mean value, standard deviation (SD) and by visual inspection. The SWT based method can remove radiometric errors effectively and preserves radiometric information to a desirable amount. From the results, SWT based method is better in smoothness and accuracy than the conventional mean filter, median filter and Discrete Wavelet Transform (DWT) based method.
\end{abstract}

Keywords: NOAA-AVHRR Images; AVHRR Sensor; Radiometric Errors; Stationary Wavelet Transform (SWT); Wavelet Thresholding.

\section{Introduction}

The National Oceanic and Atmospheric Administration (NOAA) series of polar-orbiting satellites have received significant consideration because of the on board sensor, The Advanced Very High Resolution Radiometer (AVHRR). AVHHR/3 is currently flying on NOAA $-15,18,19$, Metop-A and Metop-B. The AVHRR/3 sensor orbits the Earth at an altitude of $833 \mathrm{~km}$ and scans a width of $2399 \mathrm{~km}$. After the information of Earth's surface is recorded by the sensor, it is sent from the satellite to the ground receiving stations. AVHRR data have a spatial resolution of 1.1 square kilometers and spectral resolution of 10 bits [1]. AVHRR data have been used extensively to study about ecosystems, ice, snow, vegetation cover characteristics and land cover monitoring at global and continental scales from all over the world [2]. These applications essentially want high accuracy information.

However, sometimes AVHRR data acquisition process may be affected by several factors (senor failures, imperfectly transparent atmosphere, daily and seasonal variations in the amount of solar radiation received at the surface, imperfections in scanning instruments, signal transmission and/or decoding and atmospheric effects, etc.) that can decrease the quality and visual perception of images $[3,4]$. This may have an impact on the accuracy of the image analysis. Understandably, most users are may not satisfied with the raw data transmitted from satellites to ground stations.

The spectral data of the AVHRR sensor generally contain radiometric errors, geometric errors and atmospheric errors. Radiometric errors are caused by sensor failure and unequal detector responses. Striping, Missing lines, bad pixels or random noise is of this type [5-7]. Geometric errors are caused by satellite situa- tion such as altitude, azimuth and elevation angles, velocity, perturbations and shape of the object [8]. Image to image Misregistration and image to map Misregistration are of this group. Atmospheric errors are caused when the circumstances at the imaging time are not excellent. Cloud cover and Haze are of this class [8]. In order to use AVHRR image data effectively, error correction and enhancement methods are necessary. Therefore, they must be preprocessed separately before image analysis (land use and land cover classification, vegetation etc.).

In the literature, many researchers developed random noise correction methods for remote sensing images, mostly for Landsat TM, MODIS, Hyperspectral and CMODIS and other. The commonly used methods are mean filter, median filter [9], [10], SavitzkyGolay filter [11], frequency domain methods [12] and wavelet domain methods [13-15]. A mean filter oversmoothes and changes the details in original image. The standard median filter removes random noise and less image blur is introduced. Hybrid and adaptive median filters does not over smoothes image details and their results are better than standard median filter. A Savitzky-Golay filter will obtain a good tradeoff in image smoothing. The frequency domain approach will also suppress effectively any noise of low amplitude, but is quite helpless to remove outliers of large amplitude. Wavelet-based noisy correction methods are widely popular due to its properties such as sparsity and multi-resolution structure. These methods mostly employ non-linear thresholding of wavelet coefficients in the time-scale transform domain.

In the present work, the Stationary Wavelet Transform (SWT) is selected for removal of noise present in NOAA-AVHRR images. This method effectively maintains original information for the most part of the image. Stationary wavelet transforms decompose the image into approximation coefficients, horizontal detail coeffi- 
cients, vertical detail coefficients, and diagonal detail coefficients. Global hard thresholding is performed on horizontal, vertical and diagonal detailed coefficients by the selected threshold level. Experimental results demonstrate that the SWT based method can effectively and efficiently reduce the noisy pixels from images.

Data used here is NOAA AVHRR Level 1B collected by the receiving station installed at the Sri Venkateswara University College of Engineering (SVUCE), Tirupati (India). This ground station receives AVHRR data in HRPT (High Resolution Picture Transmission) format.

\section{AVHRR sensor and band characteristics}

The AVHRR/3 sensor is the primary imaging system flown on the NOAA POES and METOP satellites which senses passive radiation emitted from the Earth and its atmosphere. After the information of Earth's surface is recorded by the sensor, it is sent from the satellite to the ground receiving stations in real time. This data is stored in image form. Data usually has 10-bit radiometric resolution. The spatial resolution of AVHRR image is $1.1 \mathrm{Km}$, which means that one pixel at the nadir of the satellite covers an area of $1.1 \mathrm{~km} \times 1.1 \mathrm{~km}$. This sensor operates six different bands, but five bands information transmitted to ground stations simultaneously. These bands are capable of providing day and night time information about water, vegetation, clouds and the sea surface $[9,10]$. AVHRR/3 band information and its application were provided in Table 1 .

Table 1: AVHRR/3 Band Information and its application

\begin{tabular}{lllll}
\multicolumn{4}{c}{ Table 1: AVHRR/3 Band Information and its application } \\
\hline $\begin{array}{l}\text { Band } \\
\text { no }\end{array}$ & $\begin{array}{l}\text { Spectral } \\
\text { bands }\end{array}$ & $\begin{array}{l}\text { Wavelength } \\
(\mu \mathrm{m})\end{array}$ & $\begin{array}{l}\text { Resolution } \\
\text { at nadir }\end{array}$ & Band application \\
\hline 1 & $\begin{array}{l}\text { Visible } \\
\text { red light } \\
\text { Near } \\
\text { Infrared } \\
3 \mathrm{~A}, \text { Near }\end{array}$ & $0.58-0.68$ & $1.1 \mathrm{Km}$ & $\begin{array}{l}\text { Daytime cloud and } \\
\text { surface mapping }\end{array}$ \\
& $\begin{array}{l}\text { Land-water bounda- } \\
\text { ries } \\
\text { Infrared }\end{array}$ & $1.58-1.64$ & $1.1 \mathrm{Km}$ & $\begin{array}{l}\text { Snow and ice detec- } \\
\text { tion }\end{array}$ \\
3 & $\begin{array}{l}\text { 3B Infra- } \\
\text { red }\end{array}$ & $3.55-3.95$ & $1.1 \mathrm{Km}$ & $\begin{array}{l}\text { Night cloud map- } \\
\text { ping, sea surface } \\
\text { temperature } \\
\text { Night cloud map- } \\
\text { ping, sea surface } \\
\text { temperature } \\
\text { Thermal } \\
\text { Tearface tempera- }\end{array}$ \\
& $\begin{array}{l}\text { Infrared } \\
\text { ture }\end{array}$ \\
\hline
\end{tabular}

\section{Radiometric errors}

Quality of NOAA AVHRR image is reduced if the emitted or reflected electro-magnetic energy observed by the AVHRR sensor does not coincide with the energy emitted or reflected from the same object observed from the surface. The potential sources for radiometric errors are: variation in viewing angles of sensors, scanning process across the flight path of the sensor platform, variations arising from the performance of the sensor system, electronic interference from data transmission or reception equipment, sensor characteristics and imbalance of detector responses etc. which alters the radiation received by the sensor [6], [7], [8]. Therefore, image has many errors that are referred as radiometric errors. Well-known types of radiometric errors are: Striping or banding, and missing lines.

Another type of problem is random noise caused by the restrictions in the sensing and signal transmission [5], [6]. The other potential sources may be periodic drift or degradation of the detector/sensor function over the years or electronic interference between sensor/detector components. It is occurring in the image randomly. This problem largely observed in many remote sensing images (Landsat7 TM, MODIS, NOAA-AVHRR and hyperspectral images). For example, as shown in Fig.1, NOAA-AVHRR images of size $256 \times 256$ and has 8 bits radiometric resolutions contain random noise. This noise can either corrupt or totally mask the exact radiometric information hold by the image.

The data provided by them is lower in quality and also less reliable as compared to the neighboring pixels. Hence, noise removal is an essential step in remotely sensed imaging applications to highlight and recover fine details that may be hidden in the data.

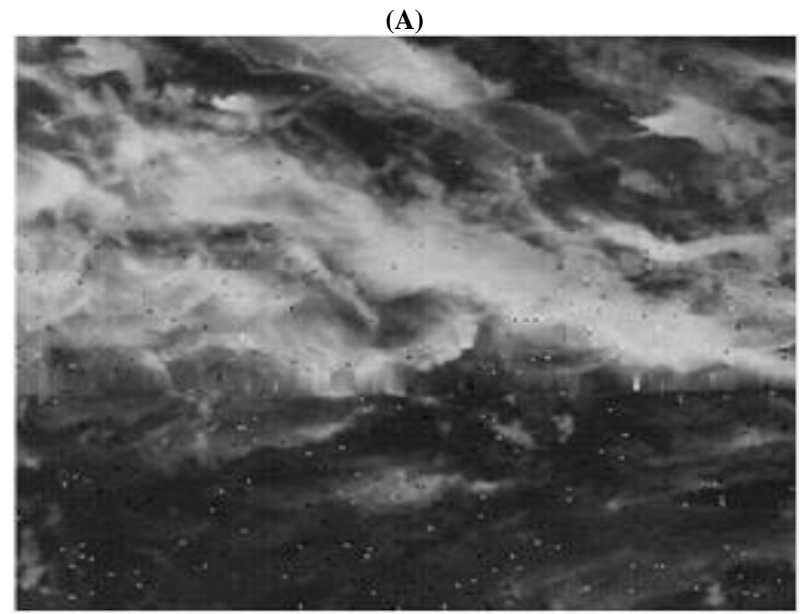

(B)

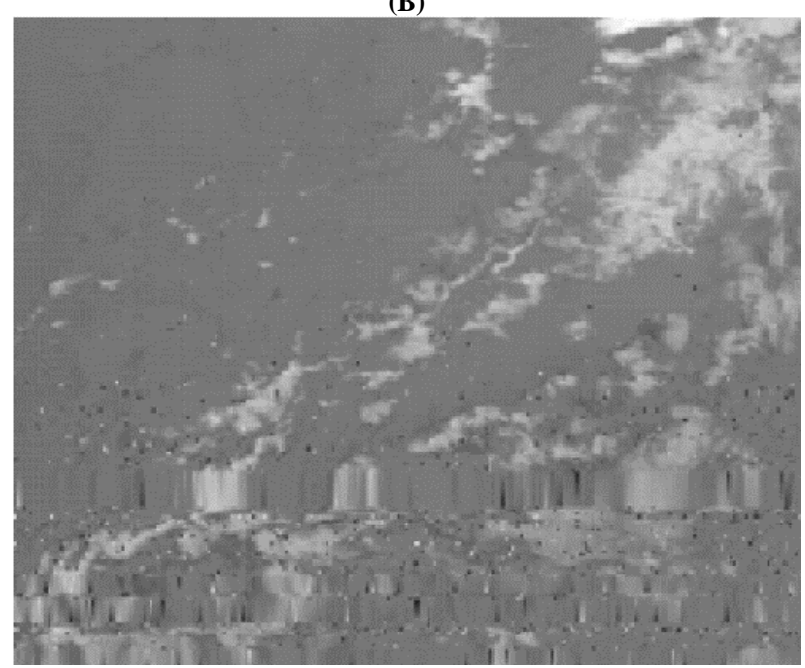

(C)

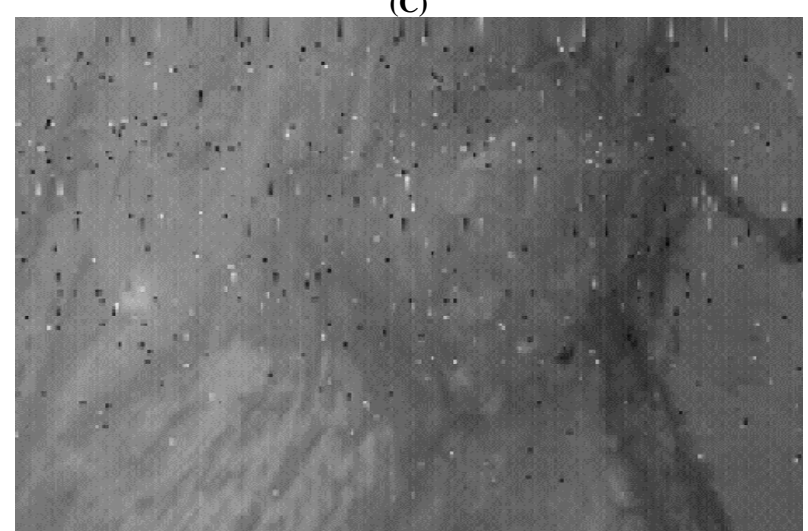

Fig. 1: NOAA 18 and NOAA 19 AVHRR Images are corrupted by random Noise, (A) 7 December 2017 NOAA 18 Image, (B) $26^{\text {th }}$ November 2017 NOAA 19 Image, (C) $7^{\text {th }}$ December 2017 NOAA 19 Image.

\section{Stationary wavelet transform (SWT)}

SWT is improved version of discrete wavelet transform. The main idea of SWT is to fill in the gaps caused by decimation step in the standard discrete wavelet transform [14], [15]. As compared to Standard DWT, SWT performs up sampling instead of down sampling [16] and Standard DWT has a time variance process between 
the wavelet co-efficient, where as SWT has a "time invariance", total number of wavelet coefficients is equal to the number of pixels in the original image after the each level of decomposition. Figure 2 describes the two level decomposition process after applying the two dimensional SWT to an image. In each level image or wavelet coefficients are divided into four sub bands as follows: approximation coefficients, vertical detail coefficients, horizontal detail coefficients and diagonal detail coefficients respectively. In Figure 2, these sub bands are denoted as $\mathrm{CA}, \mathrm{CD}^{\mathrm{V}}, \mathrm{CD}^{\mathrm{H}}$ and $\mathrm{CD}^{\mathrm{D}}$ Each sub-band size of SWT is same as the input image, so for a decomposition of $\mathrm{N}$ levels, there are redundancies of $\mathrm{N}$ in the wavelet coefficients. Information related to low frequency is concentrated on approximation coefficients and high frequency information is concentrated on detail coefficients. Noise is related to high frequencies; hence in this study threshold technique is performed on detailed coefficients during random noise correction process [17].

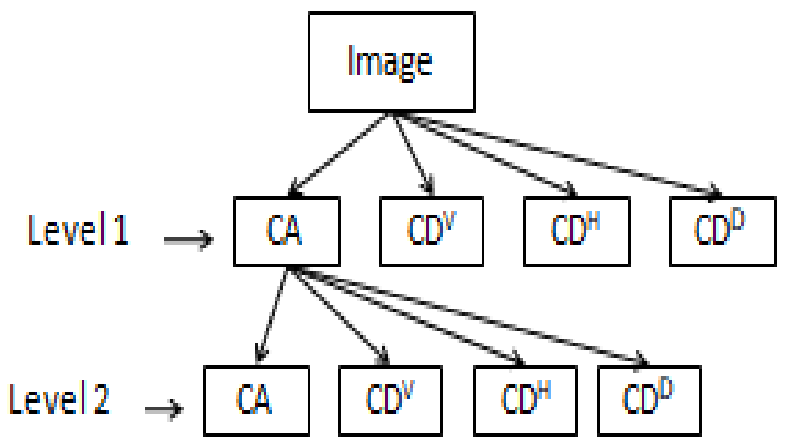

Fig.2: Two Level Stationary Wavelet Transform Decomposition.

\subsection{Proposed method}

The proposed stationary wavelet based noise removal algorithm involves five steps:

1) Forward stationary wavelet transform is performed on the noisy images. Wavelet function and level of decomposition applied to images are sym 4 and four respectively.

2) Noise variance $\sigma$ is estimated using equation (2). The threshold level is computed by equation (1).

3) Hard thresholding is performed on the detailed wavelet coefficients (Horizontal, Vertical and Diagonal based on the selected threshold level.

4) Inverse stationary wavelet transform is performed on processed wavelet coefficients to get the spatial domain enhanced image.

\subsection{Threshold selection}

Noise removal by thresholding in wavelet domain was developed first by Donoho and Johnstone. In wavelet domain, the large wavelet coefficients correspond to the data and small coefficients represents mostly noise [13]. The main problem in wavelet thresholding is to identify the finest threshold that can create a great influence on noise correction. Hence, selection of the threshold is very important in noise elimination. In this study, universal threshold is performed in the horizontal, vertical and diagonal coefficients at each level. The expression for the universal threshold is given in equation (1) as:

$$
\lambda=\sigma_{w} * \sqrt{2 * \log (L)}
$$

Where $\sigma_{\mathrm{w}}{ }^{2}$ is the noise variance and $\mathrm{L}$ is the image size.

Threshold selection requires an estimate of noise variance $\sigma_{\mathrm{w}}{ }^{2}$. Donoho and Jhonstone considered estimating noise variance in the wavelet domain and proposed a robust estimate of the noise level $\sigma_{\mathrm{w}}$ based on the Median Absolute Deviation (MAD) [20] is given in equation (2).

$$
\sigma_{w}=\frac{\operatorname{median}\{\mid(\omega k|: k=1,2,3 \ldots N|)\}}{0.6745}
$$

Here $\omega_{\mathrm{k}}$ are detailed wavelet coefficients at the finest level and ' $\mathrm{N}$ ' indicates number of detailed coefficients.

Wavelet coefficients are thresholded to the threshold level, mainly by 'hard' or 'soft' thresholding functions are illustrated in Fig. 3 the main aims of both are shrinking large wavelet coefficients and remove small ones. In hard thresholding, the decomposed wavelet coefficients below the threshold level $\lambda$ are set to zero and coefficients, which are above the threshold, are unaltered [17]. Suppose $\mathrm{X}, \mathrm{Y}$ are input and output then the hard threshold $\mathrm{T}_{h}(\mathrm{Y}, \lambda)$ is given in equation (3).

$$
\mathrm{T}_{\mathrm{h}}(\mathrm{Y}, \lambda)=\left\{\begin{array}{lr}
\mathrm{y} & \text { for } \text { all }|\mathrm{Y}| \geq \lambda \\
0 & \text { otherwise }
\end{array}\right.
$$

In soft thresholding, the wavelet coefficients are shrunk towards zero by an offset $\lambda$. It generally gives fewer artifacts and preserves the smoothness [17]. The soft thresholding function denoted as $T_{s}$ $(\mathrm{Y}, \lambda)$ and is given in equation (4) as

$$
\mathrm{T}_{\mathrm{s}}(\mathrm{Y}, \lambda)=\left\{\begin{array}{l}
\mathrm{y}-\lambda, \text { if } \mathrm{y} \geq \lambda \\
y+\lambda, \text { ify } \leq-\lambda \\
0, \quad \text { otherwise }
\end{array}\right.
$$

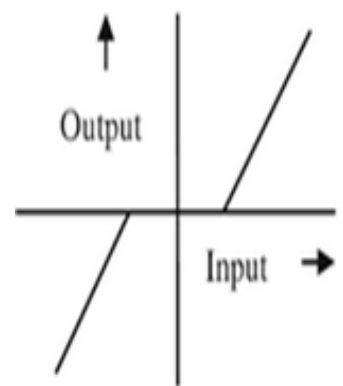

(a)

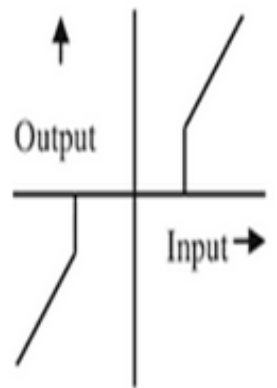

(b)
Fig. 3: (A) Soft Thresholding (B) Hard Thresholding.

\section{Results and discussions}

The reconstructed images obtained by mean filter, median filter, DWT and SWT methods for NOAA images are compared in Fig. 4, Fig. 5 and Fig. 6. It can be seen that the image for SWT method is better in the preservation of fine details and also provides good quality in visual perceptual. The results of the mean filter shown in Fig. 4 (b), [5] (b) and [6] (b) can eliminate noise effectively, but the details of the original image are also lost and the resulting image becomes slightly blurred. From Fig. 4 (c), 5 (c) and [6] (c), one can see that the median filter is capable of dissembling the noisy pixels, but it does not completely remove it. The results of DWT method are demonstrated in Fig. 4 (d), five (d) and 6 (d), can almost remove and replace noisy pixels completely, but the fine points of the original image are not better because discrete wavelet transforms do not have redundant and time-invariance. Fig. 4 (e), 5 (e) and 6 (e) shows the results of the SWT method with universal hard thresholding, which eliminates noisy pixels effectively compared with the other three methods used in this paper. Furthermore, the proposed SWT method is better in preservation of original information, fine details and shows better quality in the visual perception because of its shift invariant and redundancy. 
(A)

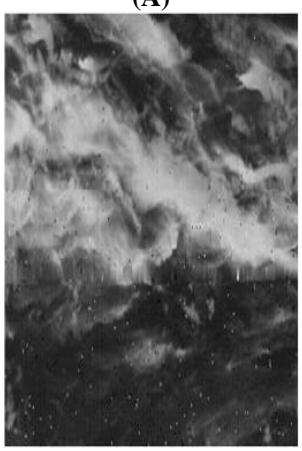

(B)

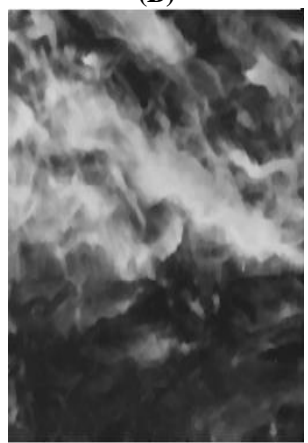

(C)

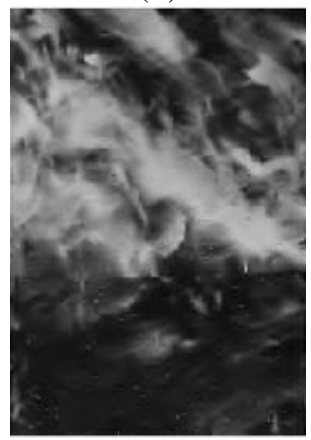

(D)

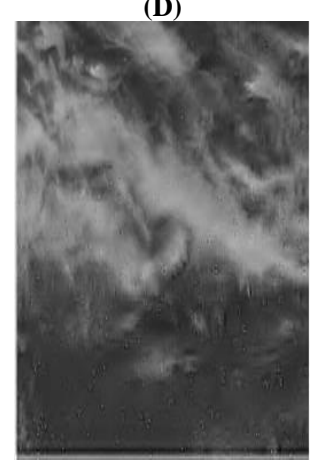

(E)

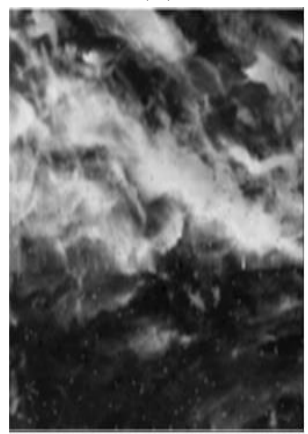

Fig. 4: (A) Original Image, Reconstructed Images Using (B) Mean Filter, (C) Median Filter, (D) DWT Method With Hard Thresholding and for Decomposition Level of four, (E) SWT Method With Hard Thresholding and for Decomposition Level of four.

(A)

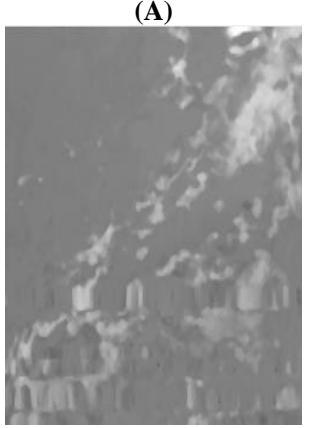

(B)

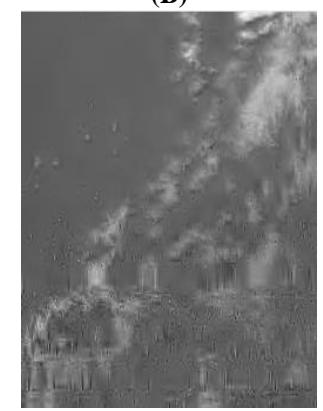

(C)

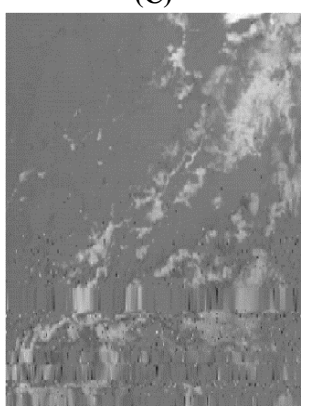

(D)

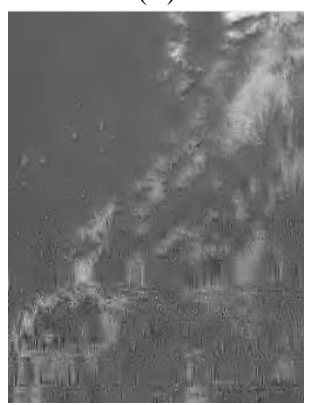

(E)

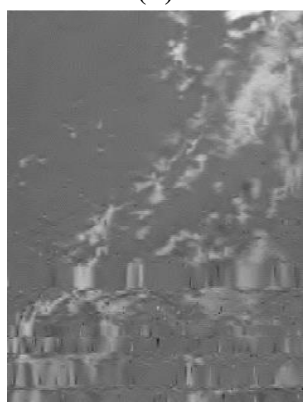

Fig. 5: (A) Original Image, Reconstructed Images Using (B) Mean Filter, (C) Median Filter, (D) DWT Method With Hard Thresholding and for Decomposition Level of four, (e) SWT Method with Hard Thresholding and for Decomposition Level of four.

(A)

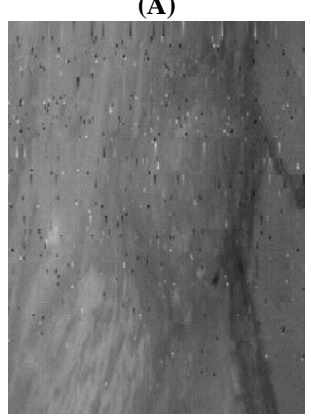

(B)

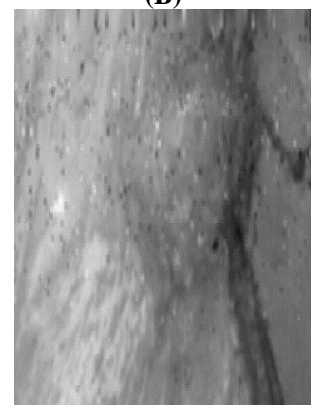

(C)

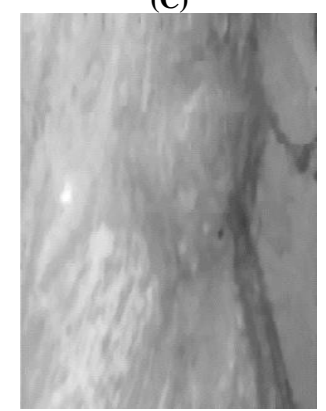

(D)

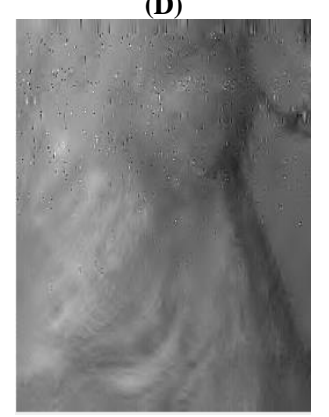

(E)

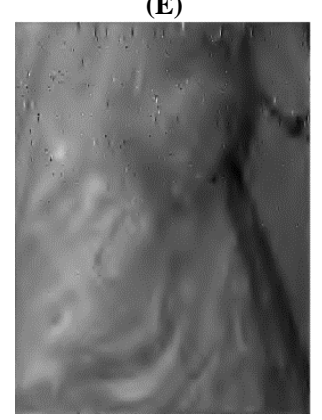

Fig. 6: (A) Original Image, Reconstructed Images Using (B) Mean Filter, (C) Median Filter, (D) DWT Method with Hard Thresholding and for Decomposition Level of four, (E) SWT Method with Hard Thresholding and for Decomposition Level of four.

\subsection{Performance evaluation}

The performance of the proposed random noise correction method is evaluated by measuring the Peak Signal-to-Noise Ratio (PSNR), Structural Similarity Index Measure (SSIM), mean and standard deviation [18],[19]. The change in the mean and standard deviation of image after removing the random noise indirectly reflects the degree of preservation of image information. Tables 2 show mean and standard deviation (SD) before and after noise correction, respectively for image 1 , image 2 and image 3 . As it is clear that, decrease in standard deviation indicates elimination of random noise from the images.

In addition, SSIM and PSNR quantities have been calculated to compare the proposed method with the conventional methods. The relations used for measuring these quantities have been shown in equations 5 and 7.

$$
P S N R=10 * \log _{10}\left(\frac{L^{2}}{M S E}\right)
$$

Here, L is a maximum intensity value of the image. When intensity values are represented by 8 bits per sample, this is 255 .
MSE is the mean square error between the reconstructed image and the original image is given in (6) by

$$
\text { MSE }=\frac{\sum_{x=0}^{M-1} \sum_{y=0}^{N-1}(\bar{f}(x, y)-f(x, y))^{2}}{M * N}
$$

Table 2: Mean and Standard Deviation for Three Test Images Before and

\begin{tabular}{|c|c|c|c|c|c|c|}
\hline \multirow{2}{*}{ Methods } & \multicolumn{2}{|c|}{ Image 1 } & \multicolumn{2}{|c|}{ Image2 } & \multicolumn{2}{|c|}{ Image3 } \\
\hline & Mean & SD & Mean & SD & Mean & SD \\
\hline $\begin{array}{l}\text { Raw } \\
\text { image }\end{array}$ & 93.99 & 53.86 & 119.84 & 17.46 & 108.47 & 23.29 \\
\hline $\begin{array}{l}\text { Mean } \\
\text { filter }\end{array}$ & 92.91 & 50.04 & 119.22 & 16.23 & 107.89 & 21.82 \\
\hline $\begin{array}{l}\text { Median } \\
\text { filter }\end{array}$ & 92.56 & 50.57 & 19.55 & 15.32 & 108.43 & 21.93 \\
\hline $\begin{array}{l}\text { DWT } \\
\text { method }\end{array}$ & 93.96 & 53.33 & 119.55 & 15.32 & 107.88 & 21.75 \\
\hline $\begin{array}{l}\text { SWT } \\
\text { method }\end{array}$ & 93.99 & 53.11 & 119.84 & 15.52 & 108.48 & 21.44 \\
\hline
\end{tabular}
after Noise Removal

The SSIM measures perceptual quality that compares patterns of pixel intensities, on the basis of contrast of the analyzed pixels [17]. SSIM index between two images $\mathrm{x}$ and $\mathrm{y}$ is defined as: 


$$
S S I M=\frac{\left(2 \mu_{x} \mu_{y}+c 1\right)\left(2 \sigma_{\mathrm{xy}}+\mathrm{c} 2\right)}{\left(\mu_{x}^{2}+\mu_{y}{ }^{2}+c 1\right)\left(\sigma_{\mathrm{x}}{ }^{2}+\sigma_{\mathrm{y}}{ }^{2}+\mathrm{c} 2\right)}
$$

Where $\mu_{\mathrm{x}}, \mu_{\mathrm{y}}$ are the average value of $\mathrm{x}$, the average value of $\mathrm{y}$. $\sigma^{2}, \sigma^{2} y$ are the variance of $\mathrm{x}$, variance of $\mathrm{y}$, and $\mathrm{c} 1=\left(\mathrm{k}_{1} \mathrm{~L}\right)^{2}, \mathrm{c} 2=$ $\left(\mathrm{k}_{2} \mathrm{~L}\right)^{2}$. Where $\mathrm{L}$ is the dynamic range of the pixel-values, and $\mathrm{k}_{1}$ and $\mathrm{k}_{2}$ are small constants to avoid instability problems when the denominator is too close to zero [17]. The results of these quantities are given in table 4 .

Table 4: PSNR and SSIM Quantities of proposed SWT and various methods

\begin{tabular}{|c|c|c|c|c|c|c|}
\hline \multirow[b]{2}{*}{ Methods } & \multicolumn{2}{|c|}{ Image1 } & \multicolumn{2}{|c|}{ Image2 } & \multicolumn{2}{|c|}{ Image 3} \\
\hline & $\begin{array}{l}\text { PSNR } \\
(\mathrm{dB})\end{array}$ & SSIM & $\begin{array}{l}\text { PSNR } \\
(\mathrm{dB})\end{array}$ & SSIM & $\begin{array}{l}\text { PSNR } \\
(\mathrm{dB})\end{array}$ & SSIM \\
\hline $\begin{array}{l}\text { Mean } \\
\text { filter }\end{array}$ & 24.068 & 0.859 & 30.258 & 0.812 & 29.615 & 0.723 \\
\hline $\begin{array}{l}\text { Median } \\
\text { filter }\end{array}$ & 24.163 & 0.828 & 31.244 & 0.799 & 30.191 & 0.705 \\
\hline $\begin{array}{l}\text { DWT } \\
\text { method }\end{array}$ & 21.381 & 0.657 & 29.857 & 0.705 & 29.168 & 0.713 \\
\hline $\begin{array}{l}\text { SWT } \\
\text { method }\end{array}$ & 32.151 & 0.867 & 34.325 & 0.878 & 30.336 & 0.733 \\
\hline
\end{tabular}

\section{Conclusions}

In this paper, random noise correction method is proposed for NOAA AVHRR images. For the DWT, SWT based methods the 'sym4' family wavelet basis and level of decomposition performed is four. The results and evaluation parameters shows that the SWT method can remove noisy pixels effectively and maintains most of the fine details. Furthermore, this technique unavoidably has more or less effect of smoothing. Proposed method depends on the selection of wavelet function and level of decomposition. Compared with mean filter, median filter and DWT method the proposed SWT method results better in preservation of fine details and visual quality of the image. Their efficiency is compared by measuring PSNR (Peak Signal to Noise Ratio), SSIM (Structural Similarity Index Measure), mean value and standard deviation (SD).

\section{Acknowledgment}

The authors are thankful to the Sri Venkateswara University College of Engineering, S.V. University, Tirupati, India for providing the data sets.

\section{References}

[1] K. Hiroshi, S. Futoki and K. Shoichi, "The AVHRR Data Processing System in the Center for Atmospheric and Oceanic Studies in the Tohoku University", Tohoku Geophysics. Journal, Vol. 34, No. 3, pp. 103-114, 1993.

[2] D. Ehrlich, J. E. Estes \& A. Singh, Applications of NOAAAVHRR $1 \mathrm{~km}$ data for environmental monitoring, International Journal of Remote Sensing, Vol. 15, Issue.1, pp. 145-161, May 2007.

[3] K. Kawano and J.I Kudoh, "Noise Line Detection Method Using Spatial Correlation of NOAA AVHRR Channels", IEEE, PP. 2377 $-2378,2006$.

[4] A.N. VAN and A. Yoshimitsu, "Error Correction for NOAA AVHRR Data Using Reference Data" Asian Association on Remote Sensing - $27^{\text {th }}$ Asian Conference on remote Sensing, ACRS, pp. 314-319, 2006

[5] A.N.Van and A.Yoshimitsu, "Precise error correction method for NOAA AVHRR image using the same orbital images", ECTI Transactions on Electrical Eng., Electronics, and Communications, Vol. 5, No. 2, pp.127-136, August 2007.

[6] W. David, "AVHRR channel-3 noise and methods for its removal", International. Journal of remote Sensing, Vol. 10, Nos. 4 and 5, pp. 645-65, 1989.

[7] J. Simpson and S. R. Yhann, "Reduction of Noise in AVHRR Channel 3 Data with Minimum Distortion", IEEE Transactions on
Geoscience and Remote Sensing, Vol. 32, No. 2, March 1994.https://doi.org/10.1109/36.295047.

[8] L. Di and Donald C. Rundquist, "A One Step Algorithm for Correction and Calibration of AVHRR Level $1 \mathrm{~b}$ Data", Photogrammetric Engineering \& Remote Sensing, Vol. 60, No. 2, pp. 165-171, February 1994.

[9] Y. Dong and S. Xu, "A new directional weighted median filter for removal of random-valued impulse noise", IEEE Signal Processing Letters, Vol. 14, pp. 193-196, March 2007.https://doi.org/10.1109/LSP.2006.884014

[10] T. Loupas, W. N. McDicken, and P. L. Allan, "An adaptive weighted median filter for speckle suppression in medical ultrasonic images", IEEE Transactions on Circuits and Systems, Vol.36, Jan 1989.

[11] Y.Liu, B. Dang, Y. Li, H. Lin, and H. Ma, "Applications of Savitzky-Golay Filter for Seismic Random Noise Reduction", ActaGeophysica, Vol. 64, No. 1, pp.101-112, Feb. 2016. https://doi.org/10.1515/acgeo-2015-0062.

[12] A. Shukla, Dr R.K. Singh, "Performance analysis of frequency domain filters for noise reduction",e-Journal of Science \& Technology, Vol. 9, No. 5, pp. 167-178, 2014.

[13] S. G. Chang, B. Yu and M. Vattereli, "Wavelet Thresholding for Multiple Noisy Image Copies," IEEE Transactions on Image Processing, Vol. 9, No. 9,pp.1631- 1635, 2000.https://doi.org/10.1109/83.862646.

[14] K. Kanagaraj, A.P. Subramonian, A. Kandasamy,“Optimal Decomposition Level of Discrete, Stationary and Dual Tree Complex Wavelet Transform for Pixel based Fusion of Multi-focused Images",Serbian Journal of Electrical Engineering, Vol. 7, No. 1, pp. 81 93, May 2010.https://doi.org/10.2298/SJEE1001081K.

[15] G. P. Nason, and B. W. Silverman, "The stationary wavelet transform and some statistical applications, " Lecture Notes in Statistics, 103, pp. 281-299, 1995.https://doi.org/10.1007/978-1-4612-2544717.

[16] R. R. Coifman and D. L. Donoho, "Translation invariant denoising" Lecture Notes in Statistics, 103, pp. 125-150, 1995.https://doi.org/10.1007/978-1-4612-2544-7_9.

[17] H. Naimi, A. B. Houda A. Mitiche and L. Mitiche, "Medical image denoising using dual tree complex thresholding wavelet transform and Wiener filter",Journal of King Saud University - Computer and Information Sciences,(2015), Vol. 27, pp. 40-45.

[18] Z. Wang, A. C. Bovik, H. R. Sheikh and E. P. Simoncelli, "Image quality assessment: from error visibility to structural similarity," Image Processing, IEEE Transactions on, Vol. 13, No. 4, pp. 600612, 2004.https://doi.org/10.1109/TIP.2003.819861.

[19] Z. Wang and A. Bovik, "A universal image quality index," Signal Processing Letters, IEEE, Vol. 9, No. 3, pp. 81-84, Mar 2002.https://doi.org/10.1109/97.995823. 